\title{
Evaluation of the incidence of inflammatory and tumor pathology of nose and nasal sinus region
}

\author{
Salah Nassrallahi1), CRistian Mircea Neagoş²), Simona Liliana Mocan3), \\ ADRIANA NEAGOŞ ${ }^{4}$ \\ 1) PhD Student, Department of Otorhinolaryngology, George Emil Palade University of Medicine, \\ Pharmacy, Science, and Technology of Târgu Mureş, Romania \\ 2) Department of General Medicine, Carol Davila University of Medicine and Pharmacy, Bucharest, \\ Romania \\ 3) Department of Pathological Anatomy, Emergency County Hospital, Târgu Mureş, Romania \\ 4) Department of Otorhinolaryngology, George Emil Palade University of Medicine, Pharmacy, \\ Science, and Technology of Târgu Mureş, Romania
}

\begin{abstract}
Inflammatory and nasal-sinus tumor pathology is a field of great interest in rhinology worldwide. The aim of the paper is to determine the prevalence of nasal and nasal-sinus inflammatory diseases, as well as nasal and nasosinusal rhinosinusitis tumors, in association or not with inflammatory diseases, using histopathological (HP) examination. It is also desired to identify the association of chronic inflammatory pathology with the tumor one, considering inflammation and immunodeficiency as local susceptibility factors. A retrospective study was performed on a group of 254 patients hospitalized between 2018-2019 in Department of Otorhinolaryngology, Emergency County Hospital, Târgu Mureş, Romania. Based on the clinical and HP examination, the distribution by inflammatory pathologies was made as follows: 175 nasal polyposis, 108 chronic rhinitis, 39 sinusitis - strictly affecting the sinus and 28 chronic polyposis rhinosinusitis - nasal and sinus association. Considering the evaluation of the incidence of benign tumor pathology, the following were found: out of the total examined cases, $4 \%$ squamous papilloma, $4 \%$ exophytic papilloma, $44 \%$ Schneiderian papilloma, $4 \%$ benign fibrous histiocytoma, $18 \%$ hemangioma, $4 \%$ hamartoma, and $4 \%$ osteoma were identified. The incidence of malignant tumors is $26 \%$ squamous cell carcinoma, $12 \%$ intestinal adenocarcinoma, $2 \%$ nonintestinal type adenocarcinoma, 2\% large B-cell lymphoma, $2 \%$ plasma cell, $2 \%$ olfactory neuroblastoma, $7 \%$ malignant melanoma, $16 \%$ basal cell carcinoma. The paper draws attention to the increased incidence of tumor and inflammatory pathology both individually and in combination, considering the involvement of the clinical correlation with the HP result completed, if necessary, with immunohistochemical examinations, for a precise diagnosis.
\end{abstract}

Keywords: rhinosinusitis, nasal polyposis, tumors, adenocarcinoma, hamartoma

\section{a Introduction}

Inflammatory nasal and nasal-sinus tumor pathology is a field of great interest in rhinology worldwide, due to its increased frequency, as well as due to the many aspects related to their etiology, contradictory clinical manifestations, and the multitude of therapeutic options.

The pathogenesis of chronic type rhinosinusal inflammatory diseases remains unclear, being much investigated. It is considered that the nasal mucosa suffers due to inflammatory phenomena, a degeneration known as polyps. They represent a hypertrophy of the mucosa because of the inflammatory processes [1]. The literature talks about the fact that these formations are produced by infectious processes, and vascular changes as local disorders of the immunity of the nasal mucosa. There are also coincidences that include allergenic factors as the cause of nasal polyposis, which are of particular interest as a constitutional factor in their genesis. There are studies in the literature that demonstrate the link between nasal polyposis and allergic diseases, analyzing different parameters of humoral and cellular immunity. An impairment of cellular immunity has been shown in the literature in patients with chronic polyposis rhinosinusitis [2].

An atypical form of chronic rhinosinusitis (CRS) is the allergic-fungal forms, characterized by the presence of eosinophils associated with fungal appearance, present in the paranasal sinuses, with or without mucosal infiltration. This condition also raises controversies about definition and etiopathogenesis. The diagnosis of this type of rhinosinusitis involves an interdisciplinary approach, including imaging, histopathology, mycology and immunological investigations, and there are controversies in the literature regarding their pathogenesis, diagnosis and treatment $[3,4]$.

Pediatric CRS is a common disorder that carries significant morbidity. The diagnosis requires sinus symptoms that persist despite standard medical therapy greater than three months. Viral infections, allergies, and anatomic differences in children lead to chronic obstruction of the ostiomeatal complex [5].

Regarding tumors, the nasal-sinus region is much less often involved. There are various histological forms of tumors and the issue of their management is raised by the proximity of the orbit and the intracranial cavities.

This is an open-access article distributed under the terms of a Creative Commons Attribution-NonCommercial-ShareAlike 4.0 International Public License, which permits unrestricted use, adaptation, distribution and reproduction in any medium, non-commercially, provided the new creations are licensed under identical terms as the original work and the original work is properly cited. 
Histopathological (HP) examinations are the most important elements in the diagnosis of tumors, they are completed where appropriate with immunohistochemical (IHC) examinations complement the HP results.

Benign tumors include more frequently of epithelial tumors than inverted papilloma, and rarer of epithelial respiratory tumors such as hamartoma, epithelial respiratory adenomatoid tumor, and seromucinous hamartoma.

Regarding zonal involvement, the literature shows that all areas of the nasal cavity and paranasal sinuses may be affected, but the lateral nasal wall, maxillary and ethmoidal sinuses are the most commonly involved. The frontal sinus and sphenoid are much less frequently the starting point, which is why they sometimes remain unknown [6]. The nasal tumors have an important role in sleep pathology together with other inflammatory pathology, as the tonsils hypertrophy [7].

Squamous cell carcinoma (SCC) is the most common form of malignancy, with a variation for each tumor type. Classical keratinizes and nonkeratinized, but with different variations, more frequently with fusiform cells or sarcomatous and lymphoepithelial. Other epithelial tumors include adenocarcinomas, olfactory neuroblastoma, melanomas, cystic adenoid carcinoma, sarcomas, chondrosarcomas, rhabdomyosarcomas, hemoproliferative tumors, and lymphomas. Rare are malignant tumors of the soft tissue, rhabdomyosarcomas, phenotypical sarcomas, and angiosarcomas $[8,9]$. There are special types of soft tissue tumors, borderline tumors, or tumors with reduced malignant potential, which develop, in this region, in special nasal glomangiopericytoma, individual fibrous tumors, and tumors with a very high local invasion and high risk of recurrence.

\section{Aim}

The aim of the paper is to determine the prevalence of nasal and nasal-sinus inflammatory diseases, as well as nasal and nasosinusal tumors, in association or not with inflammatory diseases, using HP examination. The IHC examination performed for the purpose of a definite diagnosis, came to complete the retrospective study performed. This was an exclusion criterion from the study, not being its main object. It is also desired to identify the association of chronic inflammatory pathology with the tumor one, considering inflammation and immunodeficiency as local susceptibility factors.

\section{口 Patients, Materials and Methods}

A retrospective study was performed on a group of 254 patients hospitalized between January 1, 2018 until December 31, 2019 in the Ear, Nose and Throat (ENT) Clinic, University Emergency County Hospital, Târgu Mureş, Romania. The inclusion criteria were: patients who underwent surgical procedures, subsequently HP analyzed, patients with rhinosinusal pathology, patients diagnosed with chronic polyposis inflammation of the rhinosinusal region, benign and malignant tumors.

In order to obtain fragments for HP analysis, patients underwent incisional or excisional biopsy. The written consent of the patient was obtained to perform the biopsy. For the performance of the statistics, the anonymous data of the patients were used, being fully respected the personal data.
Tissue samples were analyzed macroscopically, were processed and microscopically visualized, stages completed or not by complementary investigation, as appropriate. Hematoxylin-Eosin (HE) staining was used for histological examination of the samples.

Statistical evaluation of the data was performed on a group of 254 hospitalized patients with various nasal and nasal-sinus pathologies: chronic polyposis rhinitis, chronic polyposis sinusitis, chronic polyposis rhinosinusitis, and nasal tumor pathology, such as squamous papilloma, exophytic papilloma, Schneiderian papilloma, histiocytoma, hemangioma, hamartoma, osteoma, SCC, intestinal adenocarcinoma, basal cell carcinoma (BCC), nonintestinal type adenocarcinoma, large B-cell lymphoma, plasmacytoma, olfactory neuroblastoma, malignant melanoma. After the analysis of the demographic parameters of the lesion diagnosis, the IHC analyzes were performed.

For the study realization and cases analyzes was obtained the approval of the Ethics Commission of the University Emergency County Hospital of Târgu Mureş. All data collected were statistically analyzed using Statistical Package for the Social Sciences (SPSS) ver. 24 , for qualitative data description or percentage for quantitative evaluation and standard deviation.

\section{ㅁ Results}

Out of the total number (254) of cases, the distribution by age group was performed as follows: $10(4 \%)$ patients less than 20 years, 23 (9\%) patients between $20-29$ years, $31(12 \%)$ patients between $30-39$ years, $53(21 \%)$ patients between 40-49 years, 53 (21\%) patients between 50-59 years, $56(22 \%)$ patients between $60-69$ years and $28(11 \%)$ patients over 70 years.

The distribution by gender was made as follows: 92 (36.22\%) female patients and $162(63.78 \%)$ male patients.

Based on the clinical and HP examination, the distribution by inflammatory pathologies was made as follows: 175 patients with chronic polyposis inflammatory pathologies not associated with tumors, of which 108 (62\%) patients with chronic polyposis rhinitis, 39 (22\%) patients with chronic polyposis sinusitis - strictly affecting the sinus and $28(16 \%)$ patients with CRS with polyps nasal and sinus association (Figure 1).

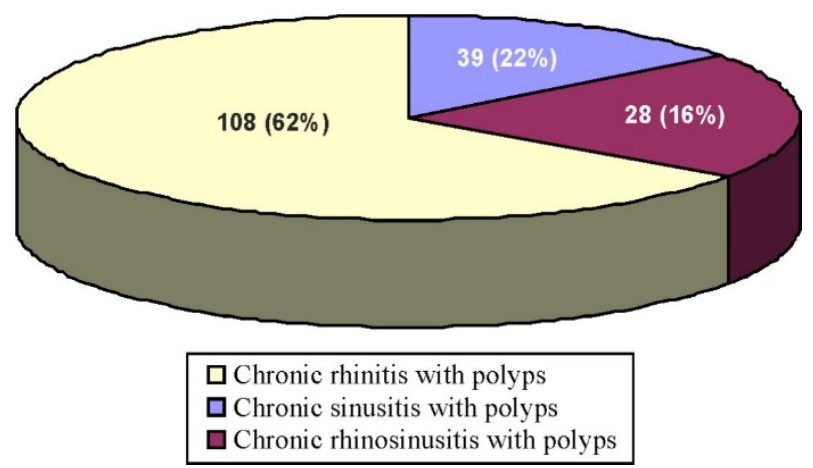

Figure 1 - Incidence of nasal and nasal sinus inflammatory pathology.

From total of 254 patients, to 175 inflammatory pathology was highlighted. The incidence of nasal and sinus tumor pathology being highlighted in 79 patients.

Benign nasal and sinus tumors occur in $64(82 \%)$ patients and malignant tumors in $15(18 \%)$ patients. 
Considering the evaluation of the incidence of benign tumor pathology, the following were found: out of the total examined cases, three (4\%) patients with squamous papilloma, three (4\%) patients with exophytic papilloma, $35(44 \%)$ patients with Schneiderian papilloma, three (4\%) patients with benign fibrous histiocytoma, $14(18 \%)$ patients with hemangioma, three (4\%) patients with hamartoma, and three $(4 \%)$ patients with osteoma were identified. The difference was in various other types of benign tumors (Figure 2).

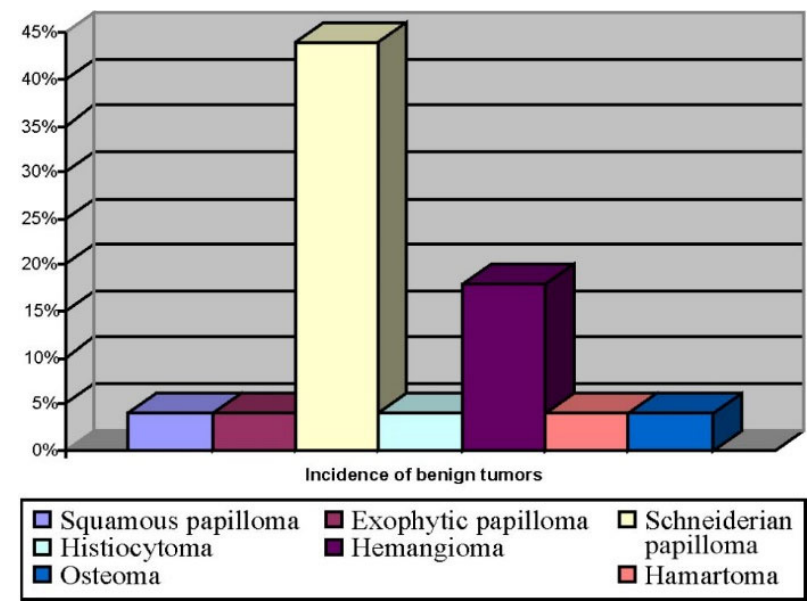

Figure 2 - Incidence of benign tumors with nasal and rhinosinusal localization.

Out of a total of 15 patients with malignancies identified over a period of one year, the incidence by tumors was as follows: six (7\%) patients with SCC, three (4\%) patients with intestinal adenocarcinoma, one patient $(1 \%)$ nonintestinal type adenocarcinoma, two $(2 \%)$ patients with olfactory neuroblastoma, and three (4\%) patients BCC with localization at the level of the nasal pyramid not being the object of our study, being still highlighted in the general statistics (Figure 3).

Inflammatory pathologies associated with different tumor types have been identified, as follows: chronic rhinosinusitis with nasal polyps (CRSwNP) associated with hamartoma tumors $40 \%$ and $60 \%$ association with

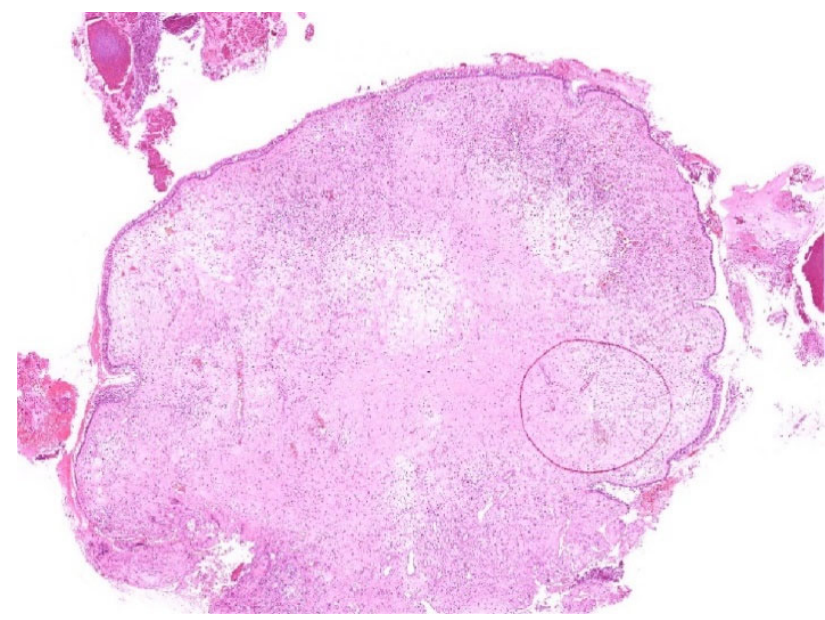

Figure 4 - Histopathological aspects of chronic inflammatory polyps - polypoid converted by respiratory epithelium, with pronounced edema in chorion, with polymorphic inflammatory infiltrate and numerous vessels, without presence of seromucinous acids. On the surface there are fragments of eosinophilic mucinous material. Hematoxylin-Eosin staining, $\times 40$.
Schneiderian papilloma, chronic polyposis sinusitis associated with tumor pathology - 33\% with osteoma, 34\% with intestinal type adenocarcinoma, 33\% with ameloblastoma (Figures 4-7).

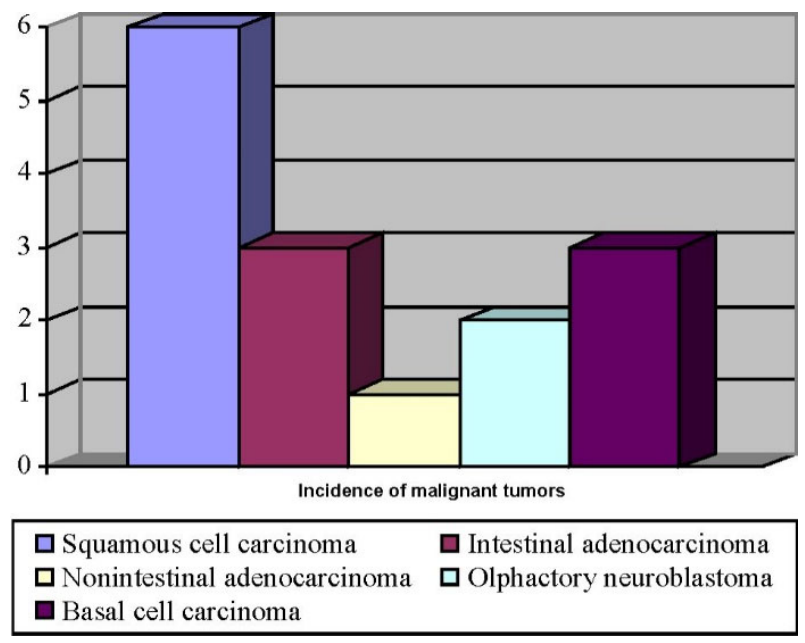

Figure 3 - Incidence of malignant tumor type diseases.

Microscopic examination of the samples taken from the formations considered to be of the inflammatory type, described the presence or absence of eosinophilic infiltrate, Charcot-Leyden crystals and the specific appearance of fungus ball in various pathologies, as follows: in chronic polyposis, microscopic polyposis, abundant eosinophilic infiltrate was described in $62.96 \%$ of cases, $13.23 \%$ of which also associated the presence of Charcot-Leyden crystals. Fungus ball has been described in $23.81 \%$ of cases, more frequently that the literature describe.

Out of the total cases, the patients diagnosed as CRSwNP, out of the total cases, $60.71 \%$ have eosinophils on microscopic examination, and $10.71 \%$ present CharcotLeyden crystals, in one case the specific appearance of fungus ball is highlighted. In chronic polyposis sinusitis, in $41.03 \%$ were described eosinophilic infiltrate, $7.69 \%$ is associated with Charcot-Leyden crystals. Statistics show as a conclusion the description of eosinophilic infiltrate in 101 cases out of 175 evaluated.

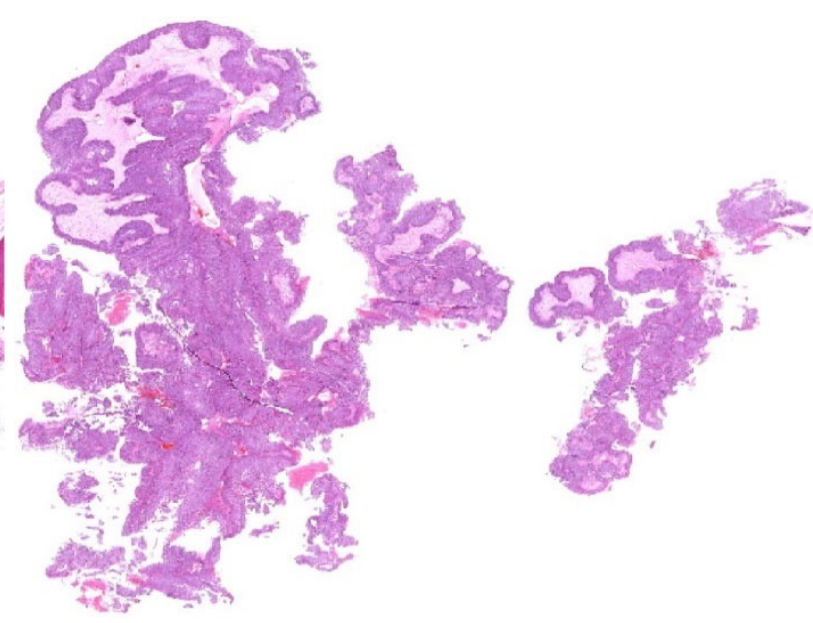

Figure 5 - Histopathological aspects of inverted Schneiderian papilloma, tumor with papillary architecture, converted by squamous epithelium, with endophytic growth. Hematoxylin-Eosin staining, $\times 40$. 


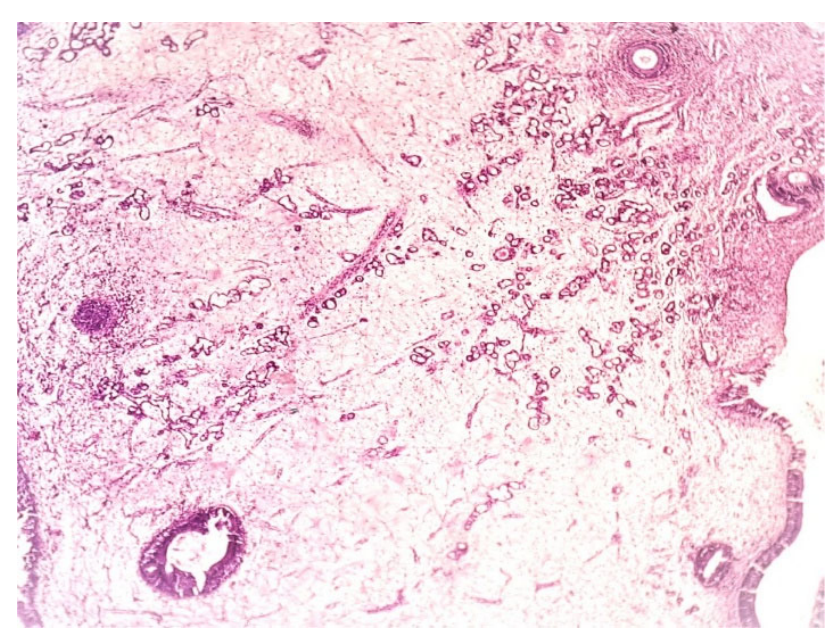

Figure 6 - Histopathological aspects of seromucinous hamartoma - fragment of mucosa with polypoid appearance, converted by respiratory epithelium, underlying with edematous chorion with chronic inflammatory infiltrate and numerous small acinar structures with round lumen delimited by a single layer of epithelial cells, without cytonuclear atypia. Hematoxylin-Eosin staining, $\times 100$.

Regarding the location of malignant tumors, $36.36 \%$ comprise the nasal region, $9.1 \%$ the sphenoid region and $9.1 \%$ in the ethmoidal region. Nasal sinus adenocarcinoma, identified predominantly in males, showed a dominant nasal and ethmoid sinus localization.

For IHC analysis, the data related to antibodies, clones, dilution, the manufacturing company were applied according to the norms of the HP examination and represent a factor of interest of the present paper.

However, we must point out that, in the examined group and evaluated clinical cases were identified the following: non-intestinal adenocarcinoma that in the IHC test was positive. Some antibodies like cytokeratin 7 (CK7), chromogranin A, synaptophysin, Melan A, S100 protein, SRY-box transcription factor 10 (Sox 10), human melanoma black-45 (HMB-45), alpha-smooth muscle actin ( $\alpha$-SMA), Factor XIIIa, cluster of differentiation (CD)20, CD68, multiple myeloma oncogene 1 (MUM1), Ki67, from DAKO Company, were used in different dilutions $(1: 200,1: 100$, 1:50, and 1:6000). Standard positive controls were used throughout, with normal serum used as the negative control.

\section{ㅁ Discussions}

It is noteworthy that most of the patients, 158 out of 254, are men aged between 40-69 years. The average age of patients is 51.26 years, with a standard deviation of 16.97 , an age range of 3-89 years. The studied group follows a normal distribution and is heterogeneous in terms of diagnostic age.

Studies in the field show similar results, both in terms of age and gender of patients. One explanation would be the fact that with age there is an immune vulnerability due to various comorbidities, and on the other hand there is a cumulative effect of inflammatory, infectious or noninfectious phenomena over time. Regarding the gender difference, it is probably due to the high prevalence of smokers over female smokers [10].

The present study shows an increased number of nasal lesions, including the nasal wings, the nasal pyramid, and

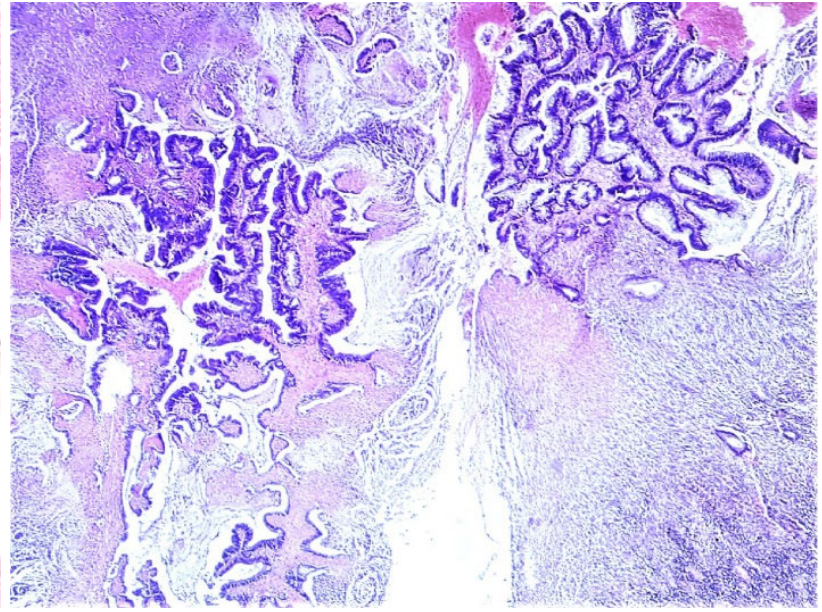

Figure 7 - Histopathological aspects of nasal intestinal adenocarcinoma, tumor with papillary architecture, with areas of ulceration and hemorrhage with exophilic growth, with conjunctival-vasculary axes converted by cylindrical stratified mucosecretory epithelium. Hematoxylin-Eosin staining, $\times 100$.

the nasal cavity itself, compared to the sinus or rhinosinusal association. BCC present located at the level of the nasal pyramid in proportion of $42.85 \%$ of the nasal pyramid, $28.57 \%$ of the nasal wings and $14.29 \%$ of the nasal vestibule was excluded from the final results, because only the nasal diseases were discussed and nasal-sinus involving the nostrils and adjacent sinus structures and do not include anything related to the structure of the nasal pyramid.

The inflammatory phenomenon was more often diagnosed than the tumor one. Of the latter, $82 \%$ were benign lesions, $18 \%$ malignant tumors were identified.

Regarding the inflammations of the nasal region and of the paranasal sinuses, they were represented in $62 \%$ by chronic polyposis rhinitis, $22 \%$ by chronic polyposis sinusitis and $16 \%$ by chronic polyposis rhinosinusitis. All have an increased prevalence in the population aged 4059 years. The pathogenesis of inflammatory pathology is related to several factors: genetic (which give background), infectious or non-infectious (exposure to various irritants). In this context, frequently discussed is CRS, which is a common condition that affects health and quality of life of patients. It is associated with inferior airway affections such as asthma, or sleep breathing disorders the involvement being controversial and much analyzed [7].

CRS is classified into two types: CRSwNP, and CRS without nasal polyps. The difference in the CRS endotype is based on the profile of inflammatory cytokines. In response to the aggression caused by inflammation, an increase in cellularity takes place locally, in order to remove harmful agents. In a study that investigated the HP profile of polyposis lesions, e.g., eosinophilic infiltrate was described in $80 \%$ of cases [11].

In our study, $55.19 \%$ of the lesions microscopically examined had described abundant eosinophilic infiltrate. Of these, one-fifth have associated Charcot-Leyden crystals, which are formed by the fusion of eosinophilic proteins. Four cases also described fungus ball. Another study showed in $86 \%$ prevalence of eosinophil cases [2]. 
Importance should be given to the type of inflammatory infiltrate. There are studies that have determined a positive association between the size of the eosinophilic infiltrate and the clinical severity of the lesion. Moreover, Arslan et al. demonstrated, in their work, that $81.8 \%$ of patients who presented eosinophilic infiltrate had postoperative recurrences, compared with $25 \%$ (who had an inflammatory infiltrate without eosinophils) [12].

The association of tumors of different types, benign or malignant with polyposis inflammatory phenomena highlighted in our study, corresponds to international multicenter studies, which show the high risk that nasal polyposis can play in triggering nasal and nasal-sinus malignancies $[12,13]$.

The definite tumor diagnosis is made following HP examination. Given the constitution of the nose and paranasal sinuses, it is understood that there is a variety of microscopic aspects, similar or not, to the tissue from which it develops, depending on the degree of differentiation of the lesion. From a HP point of view, in the tumor analysis, apart from the type of tissue, other parameters are followed, such as: invasion capacity of the tumor, constituent cells, their number, mitotic activity, different atypia, inflammatory infiltrate or associated vascularization. Lesions with a low degree of malignancy may have more numerous mitoses, which are limited to the lower third of the tissue, whose cells have different degrees of differentiation [6].

Associated investigations in both types of pathologies include computed tomography (CT), magnetic resonance imaging (MRI), which provides excellent data on bone structural details, and tumor consistency, tumor invasion in adjacent structures. Positron emission tomography (PET) $\mathrm{CT}$ is used where the tumor metastasizes to a nonspecific location relative to the primary tumor [6].

The role of all diagnostic methods, associated with each other, represent the elaboration of therapeutic behaviors that involve first of all the intranasal approach, with the preservation of the nasal anatomical structures and implicitly of the functionality of the nasal mucosa. In certain situations, related to the clinical, HP, and imaging aspects, the external surgical approach for benign and/or especially tumor resection is absolutely necessary.

When talking about the location of malignant tumors, at the nasal and sinus level, the data of our study confirm, through the values obtained $-36.36 \%$ include the nasal region, $9.1 \%$ the sphenoid region, and $9.1 \%$ in the ethmoidal region, the statements of the literature $[6,14]$. IHC analyzes are additional data in the diagnostic equation. These elements are included in the results of our study, which immunohistochemically analyzes tumors, such as melanomas, gliopericytoma, schwannomas, plasma cells, nasal neurofibromas. In this context, clinical, paraclinical data, corroborated with IHC results, lead to the possibility of a conclusive differential diagnosis. In this sense, the literature shows that although from a clinical point of view it is not very difficult to make a differential diagnosis between septal schwannoma for example and a septal neurofibroma, however, immunohistochemistry including certain specific determinations is necessary when the two clinical entities are difficult to distinguish.

The literature shows that a high-grade sinonasal carcinomas are a cohort of malignant epithelial neoplasms arising in the sinonasal cavities with distinct, ominous morphological features or lacking well-differentiated features that might otherwise classify them as less biologically worrisome. The discovery of these distinct molecular tumor profiles may have significant clinical impact as targeted molecular-based therapeutics continue to evolve, and they may offer some respite for patients who have these highly aggressive cancers [15].

\section{a Conclusions}

Chronic polyposis and/or tumor lesions of the rhinosinusal region are quite common conditions in clinical practice. Thus, the identification of etiological factors becomes an important objective, in order to successfully treat these pathologies. Regarding the objective of the present study, it was highlighted by microscopic examination of the lesions, supplemented by IHC techniques, the presence of inflammatory diseases in association with benign and malignant tumor pathology at the nasal and nasal-sinus level. Highlighting inflammatory diseases in association with benign or malignant tumor pathology is an important diagnostic element both clinically and pathologically. The paper draws attention to the increased incidence of tumor and inflammatory pathology both individually and in combination, considering the involvement of the clinical correlation with the HP result completed, if necessary, with IHC examinations, for a precise diagnosis.

\section{Conflict of interests}

The authors declare that they have no conflict of interests.

\section{Authors' contribution}

Salah Nassarallah and Adriana Neagoş have equally contribution in carrying out the study and writing the manuscript.

\section{References}

[1] Husain Q, Sedaghat AR. Understanding and clinical relevance of chronic rhinosinusitis endotypes. Clin Otolaryngol, 2019, 44(6):887-897. https://doi.org/10.1111/coa.13455 PMID: 31580530

[2] Olcott CM, Han JK, Cunningham TD, Franzese CB. Interleukin-9 and interleukin-17C in chronic rhinosinusitis. Int Forum Allergy Rhinol, 2016, 6(8):841-847. https://doi.org/10.1002/ alr.21745 PMID: 26989880

[3] Chakrabarti A, Kaur H. Allergic Aspergillus rhinosinusitis. J Fungi (Basel), 2016, 2(4):32. https://doi.org/10.3390/jof204 0032 PMID: 29376948 PMCID: PMC5715928

[4] Castillo Vizuete JA, Sastre J, Del Cuvillo Bernal A, Picado C, Martínez Moragón E, Ignacio García JM, Cisneros Serrano C, Álvarez Gutiérrez FJ, Mullol Miret J. Asthma, rhinitis, and nasal polyp multimorbidities. Arch Bronconeumol, 2019, 55(3):146-155. https://doi.org/10.1016/j.arbres.2018.09.001 PMID: 30449614

[5] Mendenhall WM, Hinerman RW, Malyapa RS, Werning JW, Amdur RJ, Villaret DB, Mendenhall NP. Inverted papilloma of the nasal cavity and paranasal sinuses. Am J Clin Oncol, 2007, 30(5):560-363. https://doi.org/10.1097/COC.0b013e3 18064c711 PMID:17921720

[6] Lund VJ, Clarke PM, Swift AC, McGarry GW, Kerawala C, Carnell D. Nose and paranasal sinus tumours: United Kingdom National Multidisciplinary Guidelines. J Laryngol Otol, 2016, 130(Suppl 2):S111-S118. https://doi.org/10.1017/S0022215 116000530 PMID: 27841122 PMCID: PMC4873911

[7] Sólyom R, Csiszér I, Neagoş A. Tonsillar hypertrophy implications in sleep disorders in children and adults. Rom J Morphol Embryol, 2014, 55(2 Suppl):603-606. PMID: 25178332 
[8] Dragonetti A, Gera R, Sciuto A, Scotti A, Bigoni A, Barbaro E, Minni A. Sinonasal inverted papilloma: 84 patients treated by endoscopy and proposal for new classification. Rhinology 2011, 49(2):207-213. https://doi.org/10.4193/Rhino09.053 PMID: 21743878

[9] Dulguerov P, Allal AS. Nasal and paranasal sinus carcinoma: how can we continue to make progress? Curr Opin Otolaryngol Head Neck Surg, 2006, 14(2):67-72. https://doi.org/10.1097/ 01.moo.0000193177.62074.fd PMID: 16552261

[10] Orb Q, Pulsipher A, Smith KA, Ashby S, Alt JA. Correlation between systemic inflammatory response and quality of life in patients with chronic rhinosinusitis. Int Forum Allergy Rhinol, 2019, 9(5):458-465. https://doi.org/10.1002/alr.22289 PMID: 30657646 PMCID: PMC6491244

[11] Alshoabi SA, Binnuhaid AA, Gameraddin MB, Alsultan KD. Histopathological analysis of sinonasal lesions associated with chronic rhinosinusitis and comparison with computed tomography diagnoses. Pak J Med Sci, 2020, 36(2):146-150. https://doi.org/10.12669/pjms.36.2.1453 PMID: 32063949 PMCID: PMC6994869

[12] Arslan F, Tasdemir S, Durmaz A, Tosun F. The effect of nasal polyposis related nasal obstruction on cognitive functions. Cogn Neurodyn, 2018, 12(4):385-390. https://doi.org/10.1007/ s11571-018-9482-4 PMID: 30137875 PMCID: PMC6048009

[13] Eschenbacher WH, Borish L. Nasal polyposis and future risk of sinonasal malignancy. J Allergy Clin Immunol, 2019, 144(4): 933-934. https://doi.org/10.1016/j.jaci.2019.08.015 PMID: 31472164

[14] Haerle SK, Gullane PJ, Witterick IJ, Zweifel C, Gentili F. Sinonasal carcinomas; epidemiology, pathology, and management. Neurosurg Clin N Am, 2013, 24(1):39-49. https:// doi.org/10.1016/j.nec.2012.08.004 PMID: 23174356

[15] Min HJ, Kim KS. Differential diagnosis between nasal septal schwannoma and nasal septal neurofibroma. J Craniofac Surg, 2017, 28(7):1780-1783. https://doi.org/10.1097/SCS.00000 00000003748 PMID: 28834833

\section{Corresponding author}

Adriana Neagoş, Senior Lecturer, MD, PhD, Department of Otorhinolaryngology, Faculty of Medicine, George Emil Palade University of Medicine, Pharmacy, Science, and Technology of Târgu Mureş, 38 Gheorghe Marinescu Street, 540139 Târgu Mureş, Mureş County, Romania; Phone +40265-215 551, Mobile +40744-112 250, e-mail: neagos.adriana@gmail.com

Received: August 26, 2020

Accepted: May 23, 2021 